\title{
Atomic Scale Modeling of Boron Transient Diffusion in Silicon
}

M. J. Caturla

A. Lilak

M.D. Johnson

M. Giles

T. Diaz de la Rubia

M. Law

Mageed Foad

This paper was prepared for submittal to the

Ion Implantation Conference

Kyoto, Japan

June 21-24, 1998

June 17, 1998

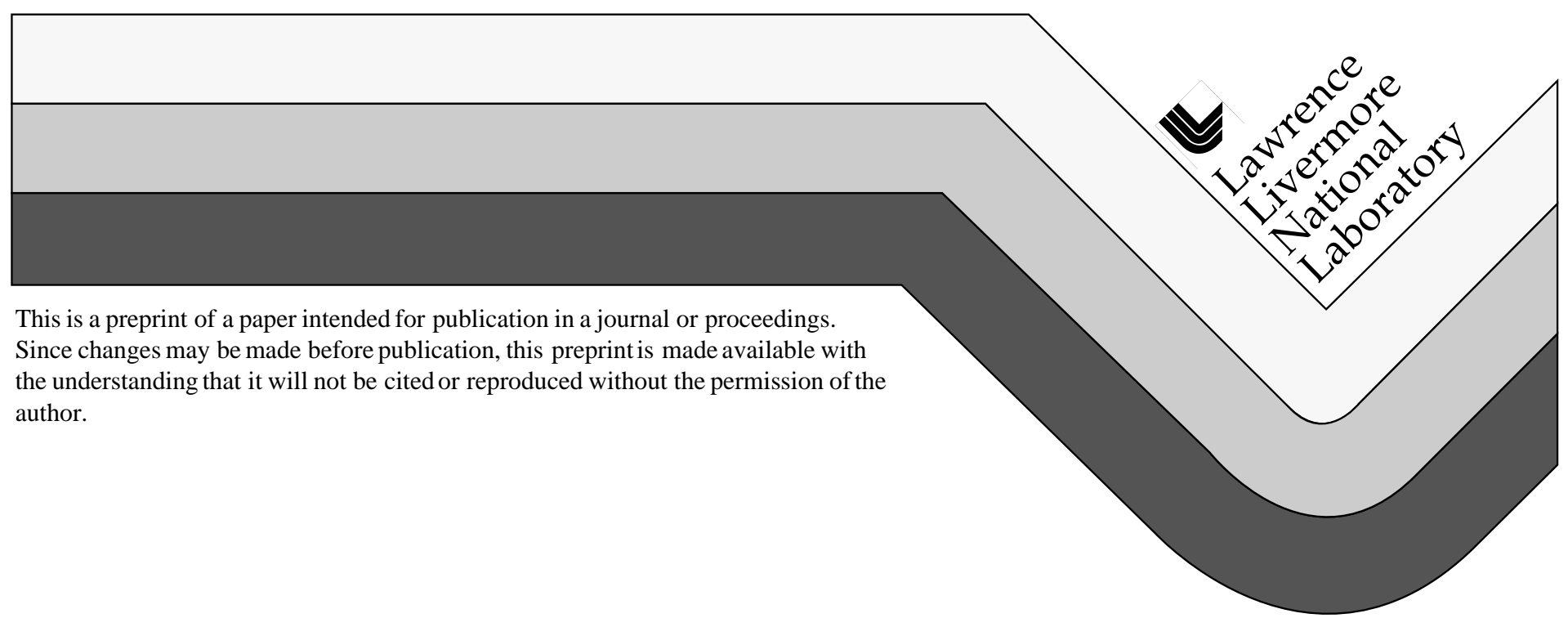




\section{DISCLAIMER}

This document was prepared as an account of work sponsored by an agency of the United States Government. Neither the United States Government nor the University of California nor any of their employees, makes any warranty, express or implied, or assumes any legal liability or responsibility for the accuracy, completeness, or usefulness of any information, apparatus, product, or process

disclosed, or represents that its use would not infringe privately owned rights. Reference herein to any specific commercial product, process, or service by trade name, trademark, manufacturer, or otherwise, does not necessarily constitute or imply its endorsement, recommendation, or favoring by the United States Government or the University of California. The views and opinions of authors expressed herein do not necessarily state or reflect those of the United States Government or the University of California, and shall not be used for advertising or product endorsement purposes. 


\title{
Atomic Scale Modeling of Boron Transient Diffusion in Silicon
}

\author{
M.J. Caturla ${ }^{1}$, A. Lilak ${ }^{2}$, M.D. Johnson ${ }^{1 *}$, M. Giles ${ }^{3}$, T. Diaz de la Rubia ${ }^{1}$, M. Law ${ }^{2}$ and \\ Mageed Foad ${ }^{4}$ \\ 1. Lawrence Livermore National Laboratory, Livermore CA 94550 \\ 2. Department of Electrical and Computer Engineering, University of Florida, Gainsville, \\ Fl \\ 3. Intel Corporation, Santa Clara, CA 95050 \\ 4. Applied Materials, Santa Clara, CA 95050
}

\begin{abstract}
We presents results from a predictive atomic level simulation of Boron diffusion in Silicon under a wide variety of implant and annealing conditions. The parameters for this simulation have been extracted from first principle approximation models and molecular dynamics simulations. The results are compared with experiments showing good agreement in all cases. The parameters and reactions used have been implemented into a continuum-level model simulator.
\end{abstract}

\section{Introduction}

Predictive modeling of silicon bulk processing requires the development of physically-based simulation tools. These tools should be capable of predicting the spatial dopant distribution after implantation and annealing with a minimum of parameter re-fitting from one processing condition to another. In this paper we present an atomistic approach to the development of predictive process simulation tools. We first discuss the use of first principles methods to construct a database of point defect, extended defect and dopant energetics. These results provide us with a thorough fundamental understanding of interstitial and vacancy diffusion kinetics, as well as of the interaction of these defects with boron and carbon. Next, we use this database as input for kinetic Monte Carlo simulation of ion implantation and dopant diffusion under a wide variety of technologically relevant conditions. We show that out simulations are in excellent agreement with experiments for $40 \mathrm{keV}$ Boron implants in silicon annealed at $700 \mathrm{C}, 800 \mathrm{C}$ and $900 \mathrm{C}$ for various lengths of time. The simulations predicts all the boron profiles with great accuracy without need for any parameter fitting. Moreover, we show how the atomistic nature of the calculations can be used to make novel prediction regarding the time evolution of the active boron fraction during annealing. Finally, we discuss how this ab initio database and kinetic Monte Carlo results can be used in conjunction with continuum-level process simulators to model silicon bulk processing, and compare the results to experiments.

\section{The model}

We have developed a kinetic Monte Carlo simulator to study the implantation and annealing of Boron in Silicon. This model is based on the work by Heinish et al. [1]. The defects, vacancies and interstitials, and dopants are represented as points with a set of conditions, such as interaction radius, size (for the case of clusters), shape and diffusivities. Interactions between dopants and defects can also be included, such as the case of Boron clustering to Silicon self-interstitials. 
Diffusivities and binding energies of clusters set up the probabilities for a process to occur, diffusion or cluster dissociation respectively. Those mobile particles move a fixed distance, first nearest neighbors in this case, in a random direction in the crystal.

The input data for such a simulation consists then on the values for diffusivities and binding energies of the different species. These values can be extracted from first principle calculations or other basic simulations. In our case we use the values for the diffusivities of vacancies and self-interstitials obtained from molecular dynamics simulations [2]. The migration path for Boron in Silicon has been calculated by Zhu $[3,4]$ and the energetics obtained are used in our simulation. Besides Boron migration, the inteaction between Boron with silicon selfinterstitials were also studied by Zhu. From these simulations binding energies of B-I complexes were obtained and are included in the kinetic Monte Carlo simulation. For details on these values see refs. [5].

In the case of ion implantation another input for the kinetic Monte Carlo simulation is the initial configuration of defects (vacancies and interstitials) and dopant (Boron). We obtain this three dimensional distribution from a binary collision code such a UT-Marlowe [6], proved to give accurate as-implanted profiles for Boron at energies above 1 $\mathrm{keV}$. Individual ions are simulated with this model to a total of at least 2000 independent cascades. The different ions are included in the Monte Carlo with a time spacing between two ions given by the dose rate of the simulation.

\section{Results}

In figure 1 we present the results for the concentration of Boron as a function of depth after implantation of $40 \mathrm{keV}$ Boron to a total dose of $2 \times 10^{14}$ ions $/ \mathrm{cm}^{2}$, and a dose rate of $10^{12} \mathrm{ions} / \mathrm{cm}^{2} / \mathrm{s}$ and at room temperature. The continuum line is the SIMS profile for the same experimental conditions. The circles show the total Boron concentration profile. The concentration of Boron clusters after the implantation are also shown in the same figure. During implantation single vacancies and interstitials are produced by the energetic ions. Vacancies are able to migrate at this low temperature, forming small clusters, mostly of two vacancies. Interstitials, on the other hand are basically inmobile. Some of them will interact with the Boron ions implanted forming BI complexes. The two complexes that dominate after the implantation is a Boron with an self-interstitial (BI) and a Boron with two interstitials (BI2), as we show in fig 1. The total number of Boron in substitutional places after implantation, that is, the Boron active is appoximately $50 \%$ of the total implanted Boron.

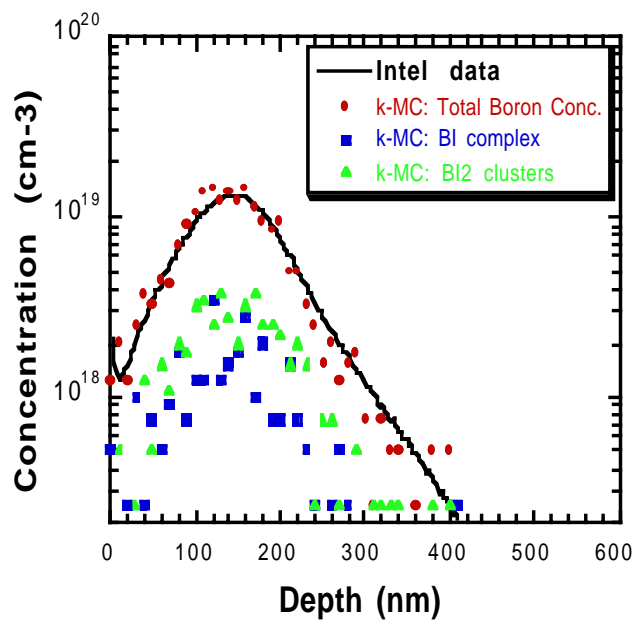

Fig 1. As-implanted Boron concentration profile for $40 \mathrm{keV}, 2 \times 10^{14} \mathrm{ion} / \mathrm{cm}^{2}$. Continuum line is SIMS data. circles are kMC simulation for the total Boron concentration. Squares and triangles are BI and $\mathrm{BI} 2$ respectively from the simulation. 
In order to remove the damage produced by the implanted ions and activate the dopant, the sample is annealed. In fig 2 we present the concentration profile for Boron after annealing at $800 \mathrm{C}$ for 60 minutes. Like in fig 1 , the line shows the results of a SIMS profile for the same conditions. The circles show the results from the kinetic Monte Carlo simulation. Observe the good agreement between the experiments and the simulations. The squares show the concentration of Boron in clusters. The presence of Boron in clusters produces the immobile peak observed in the profile, while the tail of the profile moves during annealing.

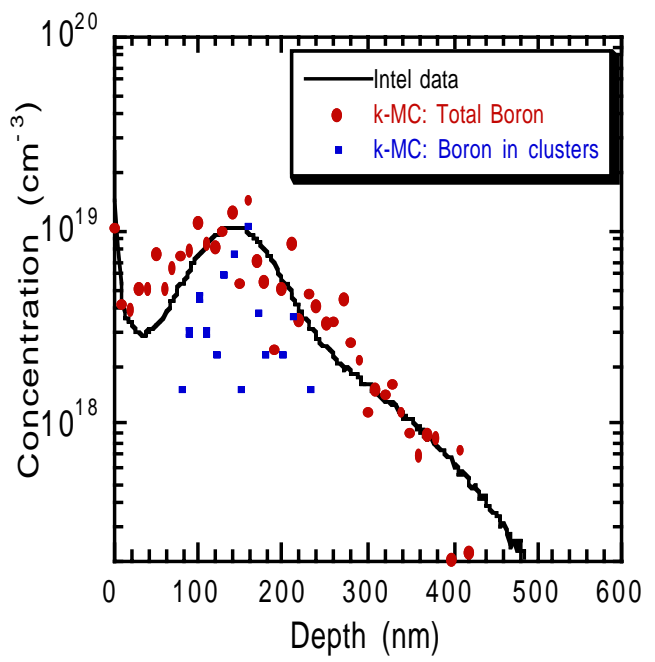

Fig 2. Boron concentration after annealing at $800 \mathrm{C}$ for 60 minutes. Line is SIMS data. Circles are total boron concentration from kMC simulations. Squares are concentration of boron in clusters, B3I, from $\mathrm{kMC}$ simulations.
Fig 3 shows the evolution of the interstitial and vacancy clusters during the annealing time. In the left axis we present the average cluster size for vacancies (dashed line) and interstitials (continuum line). On the right axis we present the percentage of Boron active in the lattice. At the beginning of the annealing clusters of vacancies grow, due to the lower diffusivity of vacancies with respect to interstitials and the lower binding energies for vacancy clusters. After $10 \mathrm{~s}$ all the vacancies have either recombined with other defects (interstitials or Boron interstitials) or with the surface. At this time, interstitial clusters can start growing, and the total number of self interstitials is equal to the implanted dose, as predicted by the +1 model [7]. At this time basically all the Boron implanted is active. As soon as vacancies dissapear from the lattice there is a higher probability for Boron interstitials, that are mobile, to recombine

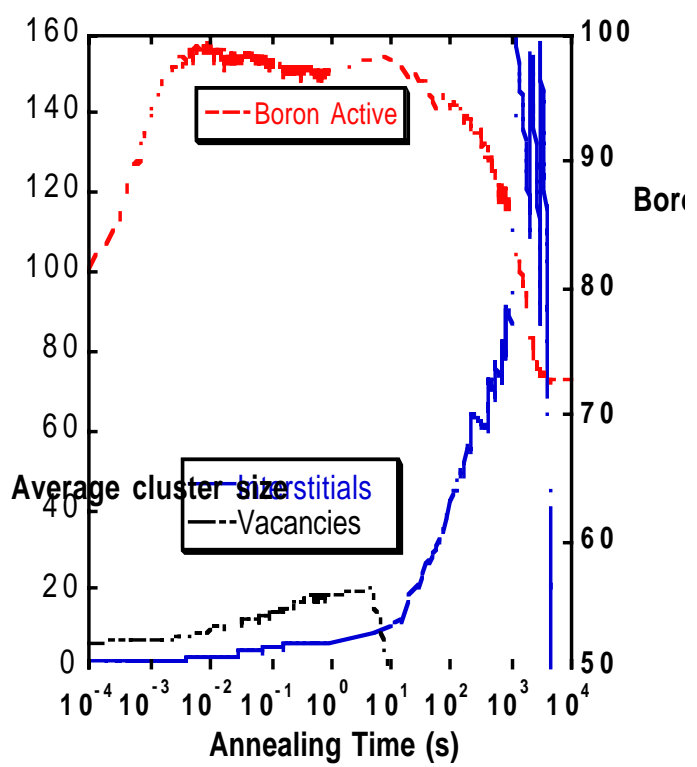

Fig 3. Defect evolution during annealing at $800 \mathrm{C}$. Right axis indicates the percentage of boron active. Left axis shows the average cluster size (solid line is interstitials, dashed lines is vacancies). with silicon self-interstitials, forming 
larger order Boron clusters. The formation of this Boron clusters decreases the number of active Boron atoms, as it can be observed in fig 3. After the annealing approximately $70 \%$ of all the implanted Boron is active, about $20 \%$ more than after implantation. Therefore, as a prediction of the model, there is a short time during which $95 \%$ of the Boron is active.

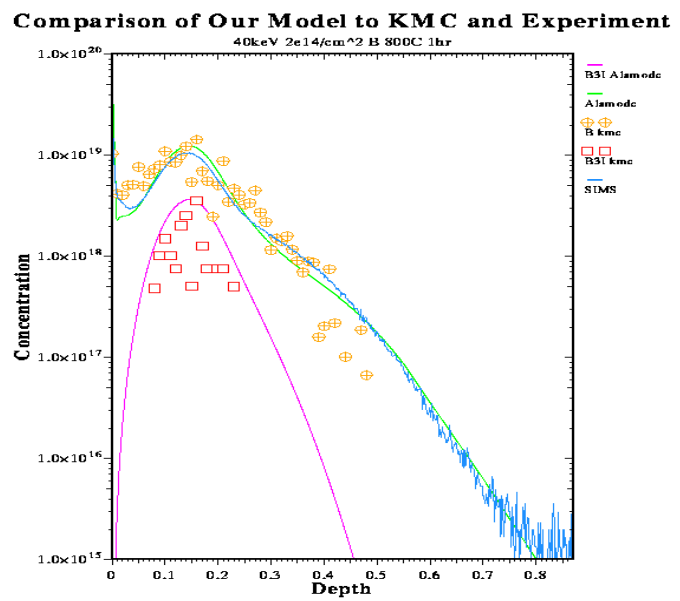

Fig 5. Comparison between Alamode simulations using the parameters obtained from $a b$ initio for Boron migration and binding energies of B-I cluster, and from molecular dynamics for Vacancy and Interstitial migration and clustering, with $\mathrm{k}$ Monte Carlo simulations and experimental profiles.

This model has been implemented into a continuum model simulation. The equation solver Alamode was used to simulate the same conditions presented here. The input data used for this simulation was the same as the one used for the kinetic Monte Carlo simulations. Also in this case good agreement was obtained both with the kinetic Monte Carlo results and the experimental data, without fitting any of the parameters of the simulation. Fig 5 shows the results of the simulation using Alamode for the case of $800 \mathrm{C}$ anneal presented in this paper. We include in this figure the comparison between Alamode, the kinetic Monte Carlo simulations and experimental data. Observe the good agreement between all three. This hierarchy of simulation methods can be of great interest for future development of predictive models for ion implantation and defect diffusion.

\section{References}

[1] H. L. Heinisch, Nucl. Instrum. and Methods, B102, 47 (1995)

[2] G. Gilmer, T. Diaz de la Rubia, M. Jaraiz, Nucl. Instrum. and Methods B102, 257 (1995)

[3] J. Zhu, T. Diaz de la Rubia, C. Maihot, Phys. Rev. B54, 4741 (1996)

[4] J. Zhu, Mater. Res. Soc. Symp. Proc. 469. 151 (1997)

[5] M. D. Johnson., M. J. Caturla, T. Diaz de la Rubia., J. Appl. Phys. (in press)

[6]. S. Tian, S. J. Morris, B. Obradovic, M. F. Morris, G. Wang, G. Balamurugan., A. F. Tasch, C. Snell, U.T, Marl;owe, Version 4.0 (1996)

[7] M. Giles, J. Electrochem. Soc. 138,. 1160 (1991)

\section{Acknowledgment}

This work was performed in part under the auspices of the US Department of Energy by Lawrence Livermore National Laboratory under contract W-7405-Eng48

*Permanent address: Avanti Corporation, Fremont, CA 